\title{
Agresiones entre Adolescentes a través del Teléfono Móvil y de Internet
}

\section{Aggressions among Adolescents through Mobile Phones and the Internet}

\author{
Sofía Buelga y Javier Pons \\ Universidad de Valencia, España
}

\begin{abstract}
Resumen. El objetivo del presente estudio fue analizar la prevalencia de agresores adolescentes (cyberbullies) a través de las nuevas tecnologías de la información y de la comunicación (Internet y teléfono móvil). También, se examinaron las diferencias de género y de curso según el tipo de agresiones tecnológicas realizadas por el adolescente. La muestra estuvo formada por 1390 adolescentes de edades comprendidas entre los 12 y los 17 años. Los resultados indicaron que casi un tercio de los adolescentes han agredido a sus iguales a través de las nuevas tecnologías en el último año. En cuanto a la duración e intensidad del acoso cibernético, se observó que éste ocurre, mayoritariamente, durante menos de un mes y con una intensidad moderada, -no superior a una vez por semana-.

En la mayoría de las agresiones tecnológicas, los chicos y los alumnos del último curso de enseñanza secundaria obligatoria son más acosadores que sus compañeros.

Palabras clave: adolescencia, agresores, cyberbullying, diferencias de género y de curso, prevalencia.
\end{abstract}

\begin{abstract}
The aim of this study was to analyze the prevalence of adolescent aggressors (cyberbullies) through modern information and communication technologies (the internet and cellular phones). The differences in gender and academic year according to the type of technological aggression were also examined. The participants were 1390 adolescents aged 12-17 years old. The results indicated that almost one third of adolescents have made an attack on their peers through new technologies in the last year. Regarding the duration and intensity of cybernetic harassment, it was observed that cyberbullying mainly occurs for less than a month and with moderate intensity -no more than once a week. For the majority of technological aggressions, boys and students in the last grade of highschool bullied more than their counterparts.
\end{abstract}

Keywords: adolescence, aggressors, cyberbullying, gender and academic year differences, prevalence.

Cyberbullying entre adolescentes: prevalencia y características de los agresores. Junto a las formas tradicionales de acoso entre iguales, ha venido surgiendo y aumentando, en los últimos años, una nueva modalidad de maltrato y de intimidación entre adolescentes. Se trata del acoso a través de las nuevas tecnologías de la información y de la comunicación que supone el uso, por parte de un individuo o grupo, de medios electrónicos, tales como teléfonos móviles, email, conversadores, redes sociales, $b \log _{s}$ y páginas web, para acosar deliberada y reiteradamente a alguien, mediante ataques personales, difamaciones u otras formas. La creciente disponibilidad de internet y de teléfonos móviles proporciona nuevas vías que algunos adolescentes utilizan para provocar daño y causar angustia en sus iguales (García-Pérez, 2011; Keith y Martin, 2005). Esta nueva forma de acoso tecnológico ha sido denominada por la literatura científica principalmente como cyberbullying (Campbell, 2005; Genta, Brighi y

Correspondencia: Sofía Buelga. Departamento de Psicología Social Facultad de Psicología Universidad de Valencia Avda. Blasco Ibáñez, 21. 46010. Valencia. Spain. E-mail: Sofia.Buelga@uv.es
Guarini, 2009; Katzer, Fetchenhauer y Belschak, 2009; Patchin y Hinduja, 2006; Smith et al., 2008; Willard, 2007), habiéndose utilizado también otros términos, como online bullying (Nansel et al., 2001) o electronic bullying (Raskauskas y Stoltz, 2007).

Este nuevo tipo de maltrato entre adolescentes ocurre en una sociedad en la cual las tecnologías tienen un papel cada vez más central en la mayoría de las actividades de la vida cotidiana, y cuyos jóvenes han crecido en un medio relacional marcado por la tecnología (Li, 2006; Oblinger y Oblinger, 2005; Tokunaga, 2010). Como afirma Walker (2010), el acoso entre iguales a través de medios tecnológicos se nos presenta como un "efecto tóxico secundario" del uso generalizado de la tecnología en nuestra sociedad. De ahí, la importancia de investigar ciertos usos inadecuados de las nuevas tecnologías, que bien manejadas, presentan enormes beneficios para el adolescente y su socialización e integración social (Subrahmanyam y Greenfield, 2008; Veen, 2003).

El cyberbullying se ha definido como una "una conducta agresiva e intencional que se repite de forma frecuente en el tiempo mediante el uso, por un individuo 
o grupo, de dispositivos electrónicos sobre una víctima que no puede defenderse por sí misma fácilmente" (Smith, Mahdavi, Carvalho, Fisher, Russell, y Tippett, 2008). Las formas de maltratar a través de las nuevas tecnologías (principalmente, con internet y el teléfono móvil) son múltiples y pueden clasificarse en varios tipos. A este respecto, Willard (2006) establece una tipología, proponiendo ocho tipos principales de conductas de acoso clasificadas según la acción realizada por el agresor: a) envío de mensajes ofensivos, increpaciones, ridiculizaciones o material pornográfico no deseado; b) envío de mensajes amenazantes y coacciones; c) difusión entre terceros de rumores difamatorios sobre la víctima; d) difusión entre terceros de información confidencial sobre la víctima o de imágenes degradantes; e) sonsacamiento de información confidencial de la víctima, haciendo que la difunda entre terceros; f) exclusión deliberada de la víctima en redes sociales; g) comunicación con terceros haciéndose pasar por la víctima para dejarla en evidencia; h) discusión airada en conversadores online, con descalificativos y agresividad verbal.

El cyberbullying comparte con el acoso tradicional la característica de ser una conducta intencional, repetida, hostil y dirigida a causar daño (Buelga, Musitu y Murgui, 2009; Katzer et al., 2009; Smith et al., 2008). Como explica Li (2007), el cyberbullying es un "viejo problema en un nuevo envase", pues reproduce situaciones que ya eran comunes entre los adolescentes en los centros escolares. De hecho, Li (2006) y otros autores como Buelga, Cava y Musitu (2010) sugieren también que los problemas de bullying en el contexto escolar se trasladan y continúan en los espacios virtuales, acentuando la relación desigual y de abuso de poder entre la víctima y el agresor. Es inherente a cualquier situación de bullying el desequilibrio de poder entre acosador y acosado, así como que éste se sienta atemorizado por la intimidación intencionada y repetida de aquél (Olweus, 2006; Rigby, 2002). Las propias características de los medios tecnológicos confieren al cyberbullying un potencial dañino aún mayor, pues se hace más fácil el acoso y se incrementa, de manera importante, el distrés psicológico de la víctima adolescente (Gradinger, Strohmeier y Spiel, 2009; Spears, Slee, Owens y Johnson, 2009; Tokunaga, 2010; Willard, 2006; Ybarra, 2004), convirtiéndose esta modalidad en un problema emergente de salud pública (Bickham y Rich, 2009; David-Ferdon y Feldman, 2007; Kowalski y Limber, 2007; Monks et al., 2009).

En efecto, el mencionado desequilibrio de poder es muy elevado en el cyberbullying, pues el agresor controla la situación aun sin tener que exponerse ante su víctima. El anonimato que el medio tecnológico permite, facilita la conducta hostil e incrementa la experiencia de humillación en la víctima (McKenna, 2007; Monks et al., 2009; Walker, 2010). Tal sentimiento de indefensión y vulnerabilidad se hace más patente al no existir lugares seguros para evitar las ciberagresiones, pues la víctima no podrá huir, defenderse o evitar el acoso cuando esté recibiendo constantemente mensajes en su móvil u ordenador, o cuando esté siendo repetidamente difamada en cualquier espacio público de la red (Katzer et al., 2009; Kowalski y Limber, 2007; Smith, Mahdavi, Carvalho y Tippett, 2006). Además, la difusión entre terceros de contenidos vejatorios puede alcanzar rápidamente una audiencia muy grande de espectadores -generalmente, otros adolescentes-, aspecto que también lo diferencia del acoso tradicional cara a cara (Bickham y Rich, 2009; Huesmann, 2007). Estos espectadores pueden visionar la agresión un número indefinido de veces, pues los mensajes o imágenes difundidos en internet o por teléfono móvil pueden ser recuperados por la audiencia una y otra vez, lo cual hace que el impacto de la agresión permanezca en el tiempo, ampliándose los efectos dañinos sobre la víctima (Mora-Merchán, 2008).

Un interés creciente en la investigación actual consiste en estudiar la prevalencia del cyberbullying, (Burgess-Proctor, Patchin y Hinduja, 2009; Garaigordobil, 2011; Kowalski, Limber y Agatston, 2010; Raskauskas y Stoltz, 2007; Schultze-Krumbholz y Scheithauer, 2009), aunque la variación entre trabajos es muy notable. En efecto, la incidencia del cyberbullying varía de un estudio a otro, entre el 5\% y el $34 \%$ (Buelga et al., 2010; David-Ferdon y Feldman, 2007; Defensor del Pueblo, 2007), lo que dificulta considerablemente la comparación entre estudios tanto en un mismo país como entre países diferentes. Las razones que explican estas diferencias se deben a que no existe todavía un acuerdo con respecto a la propia definición de cyberbullying, ni tampoco con respecto a las variables medidas -incluyendo el medio de acoso y el rol de los sujetos: víctima, acosador, víctima/acosador-, a la forma de operativizar esas variables, ni con los instrumentos utilizados. Muchos trabajos evalúan la ocurrencia del cyberbullying de una forma genérica, sin especificar el tipo de agresión electrónica realizada (Calvete, Orue, Estévez, Villardón y Padilla, 2010). En todo caso, aun con estas limitaciones que deben tenerse en cuenta, son necesarias nuevas investigaciones que estudien con rigor la prevalencia del cyberbullying, que muestra ser un creciente problema mundial de los países desarrollados (Kowalski et al. 2010).

Ciertamente, en el estudio pionero de Finkelhor, Mitchell y Wolak (2000) en los Estados Unidos, la prevalencia de adolescentes acosados a través de inter-net era del 6\%. Sin embargo, en un informe posterior, estos mismos investigadores destacan un incremento del $50 \%$ en los casos de acoso tecnológico entre los años 2000 y 2005 (Wolak, Mitchell y Finkelhor, 2006). Por su parte, Smith et al. (2006) constatan que el $22.2 \%$ de adolescentes ingleses han sido víctimas de cyberbullying en los dos últimos meses, con un 5.5\% de adolescentes acosados de forma severa con una intensidad de una vez por semana o más. En España, Buelga, Cava y Musitu (2010) encuentran en un estu- 
dio reciente con 2101 adolescentes españoles, un $24.6 \%$ de adolescentes que han sido acosados por el móvil en el último año, y un $29 \%$ por internet, siendo la duración de este acoso para la mayoría de estas víctimas, menor a un mes. Estos últimos autores, como Li (2007) y Navarro (2009), observan que internet es el medio tecnológico más utilizado para agredir a los iguales. Cuando la duración del acoso se prolonga en el tiempo, tanto internet como el teléfono móvil (Buelga et al., 2010; Katzer et al., 2009) son utilizados para acosar a los iguales. La investigación de Ortega et al. (2008) establece que la modalidad más frecuente de cibervictimización es a través de conversadores como chats o Messenger, con un $14.8 \%$ de adolescentes victimizados entre los encuestados, seguido con un $4.3 \%$ por mensajes telefónicos, un $2.8 \%$ correo electrónico, un $2.7 \%$ llamadas al teléfono móvil, un $1 \%$ difusión de imágenes y vídeos en el móvil, y un $0.4 \%$ en páginas web.

Por otra parte, con respecto a los agresores de cyberbullying, los trabajos que han analizado esta cuestión son todavía muy escasos (Kowalski et al. 2010). En el estudio de Smith et al. (2006), los autores señalan que un $25.3 \%$ de los adolescentes manifiesta haber realizado uno o dos actos de agresión por internet, y un $16.6 \%$ por móvil, en los dos últimos meses, mientras que, en un acoso de tres o más veces en ese periodo, los porcentajes de adolescentes implicados son $4.4 \%$ a través de internet y $3.3 \%$ por teléfono móvil. En otra investigación, Schultze-Krumbholz y Scheithauer (2009) concluyen que un $15.5 \%$ de los adolescentes han acosado a través de internet, y un $8.5 \%$ por el teléfono móvil, en ambos casos en una medición de al menos tres veces al mes. En España, Calvete et al. (2010) encuentran que el $44.1 \%$ de los adolescentes ha realizado alguna conducta de cyberbullying. Las conductas de ciberacoso más frecuentes son apartar intencionalmente a un compañero de un grupo online (20.2\%), escribir bromas, rumores, chismes o comentarios para poner en ridículo a un compañero en internet (20.1\%), y usurpar la identidad de la víctima para crearle problemas $(18.1 \%)$.

Por lo que respecta a las relaciones entre cyberbullying y edad, las investigaciones sugieren que la etapa más crítica de victimización es la adolescencia temprana, observándose una disminución de estos comportamientos en la adolescencia media (Kowalski y Limber, 2007; Li, 2006; Smith et al., 2008; Williams y Guerra, 2007; Worthen, 2007). En este sentido, Buelga et al. (2010) encuentran en su estudio, una mayor incidencia de victimización por acoso tecnológico en los dos primeros cursos de enseñanza secundaria obligatoria, con una disminución del cyberbullying en el ciclo superior de enseñanza secundaria. En esta misma dirección, trabajos científicos realizados en España, como los del Defensor del Pueblo (2007) y los estudios de Cava, Buelga, Musitu, y Murgui, (2010) sugieren que, en general, el bullying entre adolescentes desciende pro- gresivamente con la edad, siendo más destacada la disminución de victimas y de agresores en los dos últimos cursos de Enseñanza Secundaria Obligatoria.

Sin embargo, los datos no están tan claros como en la victimización y cibervictimización, en lo que respecta a los agresores de cyberbullying. De acuerdo con Williams y Guerra (2007), la mayor incidencia de ciberagresores se sitúa aproximadamente en la edad de los 13 años. Por su parte, Ortega et al. (2008) no encuentran diferencias significativas entre edades en los roles de agresores y víctimas, aunque sí encuentran una ligera tendencia que indica que la mayoría de los ciberagresores están en los dos últimos cursos de Educación Secundaria Obligatoria mientras que la mayor parte de las cibervíctimas están en los dos primeros cursos de secundaria. Por otra parte, Calvete et al. (2010) constatan que la mayor prevalencia de agresores electrónicos está en segundo y tercer curso de enseñanza secundaria obligatoria; entre los 13-15 años. Por otro lado, Garmendia, Garitaonandia, Martínez-Fernández y Casado (2011) sugieren que mientras que el ciberacoso severo (más de una vez, por semana) es más frecuente en la adolescencia temprana, el ciberacoso de intensidad moderada (menos de una vez por semana) lo es, en la adolescencia media.

En cuanto a las diferencias de género en el cyberbullying; los pocos trabajos realizados hasta el momento tampoco son coincidentes en sus resultados. Algunos estudios señalan que no hay diferencias en la cibervictimización entre sexos (Didden et al., 2009; Hinduja y Patchin, 2008; Juvoven y Gross, 2008; Katzer et al., 2009), mientras que otros encuentran más víctimas entre las chicas que entre los chicos (Buelga et al., 2010; Burgess-Proctor et al., 2009; Dehue, Bolman y Vollink, 2008; Kowalski y Limber, 2007; Ortega et al., 2008). En relación a los cyberbullies, parece haber más acuerdo en la literatura, en indicar una mayor prevalencia de agresores varones (Li, 2006; Navarro 2009). En esta línea, Sourander et al. (2010) indican que el $16 \%$ de chicas son acosadas por chicos y sólo el $5 \%$ de los chicos son agredidos por chicas. Esa misma tendencia hacia una mayor incidencia de chicos en el rol de agresores severos y moderados es resaltada por Ortega et al. (2008). Por otro lado, Calvete et al. (2010) indican que los chicos con respecto a las chicas cometen más ciberagresiones relacionadas con las conductas de grabar y difundir imágenes degradantes sobre la víctima, así como en enviar contenido sexual no deseado y molesto.

También existen diferencias de género en cuanto a la intensidad de las agresiones cibernéticas, de modo que el ciberacoso severo (más de una vez a la semana) es realizado más frecuentemente por los chicos, y el acoso moderado (menos de una vez a la semana) por las chicas (Garmendia et al., 2011). Garmendia et al. (2011) indican que el 2\% de los chicos y el 1\% de las chicas realizan agresiones cibernéticas más de una vez a la semana, mientras que, con una frecuencia de una o 
dos veces al mes, los porcentajes son el $2 \%$ de los chicos y el $4 \%$ de las chicas. Del mismo modo, Kowalski et al. (2010) señalan que un $14 \%$ de las chicas y un $10 \%$ de los chicos han acosado a sus iguales de forma moderada durante los dos últimos meses a través de internet, mientras que el $1,1 \%$ de chicas y el $2 \%$ de chicos han agredido de forma severa.

Teniendo en cuenta estos antecedentes, el objetivo del presente estudio ex post facto fue analizar la prevalencia de agresores de cyberbullying, en una muestra representativa de alumnos españoles de Educación Secundaria Obligatoria. Se estudiaron también las diferencias de género y de curso según el tipo de agresiones cibernéticas realizadas por el adolescente.

Debido a la novedad del fenómeno del cyberbullying, el presente trabajo contribuye a ampliar el conocimiento que se tiene sobre este problema emergente en nuestro país. Se trata de un problema social que suscita una creciente preocupación en la comunidad científica, y que responde a los nuevos tiempos de una sociedad que cada vez hace un mayor uso de las nuevas tecnologías (Oblinger y Oblinger, 2005), siendo los adolescentes los protagonistas de esta nueva generación red.

\section{Método}

\section{Participantes}

La selección de los participantes se realizó mediante un muestreo estratificado por conglomerados (Santos, Muñoz, Juez y Cortiñas, 2003). Las unidades de muestreo fueron los centros educativos, públicos y concertados, de Educación Secundaria Obligatoria de la Comunidad Valenciana. Los estratos se establecieron en función de las variables: curso $\left(1^{\circ}, 2^{\circ}, 3^{\circ}\right.$ y $4^{\circ} \mathrm{de}$ la ESO) y provincia (Alicante, Valencia y Castellón). El tamaño de la muestra con un error muestral de \pm $2.7 \%$, nivel de confianza del $95 \%$ y $p=q=0.5,(N=$ 189.679), se estimó en 1308 alumnos.

La muestra estuvo formada por 1390 adolescentes de ambos sexos (740 chicos y 650 chicas) de edades comprendidas entre los 12 y 17 años $(M=13.9$ meses; $D T=1.40)$, pertenecientes a 8 centros de Educación Secundaria Obligatoria seleccionados aleatoriamente en cada una de las provincias. Un $27.2 \%$ de la muestra cursaba estudios de $1^{\circ}$ de Educación Secundaria Obligatoria (ESO), un $29.5 \%$ estaban en $2^{\circ}$ de ESO, un $20.0 \%$ estudiaban $3^{\circ}$ de ESO y por último, un $23.3 \%$ de los participantes cursaban $4^{\circ}$ de ESO.

\section{Instrumentos}

Escala de ciberagresiones. Esta escala está formada por 10 ítems (ver tablas 2 y 3) que evalúan comportamientos que implican agresiones de hostigamiento, persecución, denigración, violación de la intimidad, exclusión social y suplantación de la identidad. La escala mide con un rango de respuesta de 1 a 5 (nunca, pocas veces: entre 1 y 2 veces, algunas veces: entre 3 y 5 veces, bastantes veces: entre 6 y 10 veces y muchas veces: más de 10 veces), las agresiones cometidas por el adolescente durante el último a través las nuevas tecnologías de la información y comunicación (teléfono móvil y Internet). El coeficiente de fiabilidad $\alpha$ de Cronbach en el presente estudio es de 0.88 .

Intensidad de ciberagresiones. La intensidad del ciberacoso durante el último año se evalúa con 5 opciones de respuesta que incluyen: nunca, 2 ó 3 veces, 1 ó 2 veces al mes, 1 ó 2 veces a la semana y todos o casi todos los días. Las dos primeras modalidades de respuesta de intensidad permiten evaluar el acoso moderado (menos de una agresión por semana) y las dos últimas el acoso severo (más de una agresión por semana) (Smith et al., 2006).

Duración de ciberagresiones. La duración del acoso tecnológico durante el último año se evalúa con 5 opciones de respuesta: nunca, 1 mes (o menos), entre 3 y 6 meses, entre 6 y 12 meses, y un 1 año (o más).

\section{Procedimiento}

Se solicitó permiso por carta a cada uno de los centros docentes seleccionados aleatoriamente para participar en la investigación. Una vez obtenidos los permisos, se realizó un seminario informativo con la dirección y profesorado para explicarles los objetivos de la investigación y se obtuvieron las autorizaciones paternas. Investigadores previamente entrenados realizaron durante el horario escolar la aplicación de los instrumentos de forma individual y anónima.

\section{Análisis de Datos}

Para analizar la prevalencia de agresores (cyberbullies) a través de las nuevas tecnologías de la información y comunicación se realizaron análisis de contingencia y se calculó el coeficiente de correlación de Pearson en las variables objeto de estudio. Se agrupó en el cuestionario de intensidad del acoso el porcentaje de respuestas dadas en las variables « 2 ó 3 veces » y « 1 ó 2 veces al mes» en la variable agresión moderada (menos de una agresión a la semana) y el porcentaje de respuestas obtenidas en las variables « 1 ó 2 veces a la semana» y 〈〈todos o casi todos los días» en la variable agresión severa (más de una agresión a la semana).

Posteriormente, se realizó un análisis multivariante de la varianza, MANOVA 2 X 4, entre sexos (varones, mujeres) y cursos $\left(1^{\circ}, 2,3,4^{\circ}\right.$ de ESO) en las variables de ciberagresiones. Al no hallarse diferencias estadísticamente significativas en la interacción entre sexo y curso $(\Lambda=.97 ; F(6,4030)=1.28, p>.05)$, se analizaron los efectos principales univariados de los dos factores. En 
los casos donde se observaron diferencias significativas en el efecto principal del curso, se aplicaron pruebas post hoc mediante el procedimiento de Bonferroni.

\section{Resultados}

\section{Prevalencia de cyberbullies}

Los resultados indican que el $68.6 \%$ ( $n$ 954) de la muestra total nunca ha agredido a sus iguales a través del teléfono móvil y de Internet. Un tercio de los adolescentes de la muestra, el 31.4\% $(n=436)$, sí que ha acosado a sus pares en el último año. El acoso realizado por la mayoría de estos adolescentes a los iguales dura menos de un mes $(24.1 \%, n=335)$, siendo las agresiones de intensidad moderada $(22.1 \%, n=307)$. Se observa, por tanto, un descenso muy significativo en la prevalencia de adolescentes que han agredido a sus iguales más de un mes. Un $4.3 \%$ de estos adolescentes $(n=60)$ han acosado entre 3 y 6 meses, un $2.6 \%$ $(n=36)$ entre 6-12 meses, y un 0.4\% $(n=5)$ más de un año.

En cuanto a la intensidad del acoso, el 26.5\% $(n=$ 368 ) de los agresores acosan de forma moderada mientras que el $4.9 \%(n=68)$ lo hace de forma severa. A este respecto, los datos indican que el $2.0 \%(n=28)$ de agresores severos han acosado a sus iguales durante un mes o menos, un $1.4 \%(n=20)$ entre 3 y 12 meses, un $1.2 \%(n=16)$ entre 6 y 12 meses, y un $0.3 \%(n=4)$ más de un año.

Se observa también que existe una relación significativa entre la intensidad de las agresiones y la duración de las mismas, de modo que la duración del acoso cibernético se relaciona con una intensidad más elevada de ciberagresiones $(r=.85, p<.01)$.

\section{Diferencias entre sexos y curso en el tipo de ciberagresiones}

Los resultados revelan que existen diferencias esta- dísticamente significativas entre chicos y chicas (tabla 2). De las 10 ciberagresiones, los resultados indican que los chicos puntúan significativamente más alto que las chicas en la mitad de las agresiones, no existiendo diferencias en el resto de las agresiones.

Los chicos tienen puntuaciones estadísticamente más altas que las chicas en todas las conductas de hostigamiento y de persecución. Las conductas de hostigamiento son el tipo de agresión dónde las diferencias son estadísticamente mayores entre sexos $\ll$ He insultado o ridiculizado a alguien con mensajes o llamadas〉> $(F(1,1390)=12.12, p<.001), \mathrm{y}\langle$ He dicho o enviado cosas de miedo o cosas guarras para asustar o fastidiar a alguien $>(F(1,1390)=9.13, p<.005)$. También existen diferencias significativas entre chicos y chicas en las conductas de persecución « cosas con amenazas $>(F(1,1390)=4.50, p<.05)$, y «He amenazado para meter miedo» $(F(1,1390)=$ $6.44, p<.05)$. En las agresiones de violación de intimidad, los datos indican también que los chicos puntúan más alto que las chicas en la agresión «manipular fotos o videos de alguien sin su permiso»> $(F(1,1390)$ $=5.50, p<.05$ ).

En las demás ciberagresiones, aunque la tendencia señala que los chicos puntúan más alto que las chicas en todas las agresiones, excepto en exclusión social dónde las chicas tienen puntuaciones medias más elevadas que los chicos, los resultados muestran que no hay diferencias estadísticamente significativas entre sexos.

En cuanto a las diferencias entre cursos, los datos indican que el curso $4^{\circ}$ de la ESO es el curso que obtiene puntuaciones estadísticamente más elevadas en 7 de las 10 ciberagresiones evaluadas. Las diferencias se hallan, principalmente, entre este último curso de Educación Secundaria Obligatoria y el curso $3^{\circ}$ de la ESO (tabla 3). Hay diferencias significativas entre $4^{\circ}$ y $3^{\circ}$ en la agresión de denigración « $\ll$ He contado mentiras o rumores falsos sobre alguien〉 $(F(3,1390)=2.70, p$ $<.05$ ), y en las 3 agresiones de violación de la intimidad. Así, en violación de la intimidad, el curso $4^{\circ}$ de la ESO puntúa estadísticamente más alto que el curso $3^{\circ}$ de la ESO en las conductas « $\mathrm{He}$ contado secretos de

Tabla 1 Incidencia de la intensidad y duración de las ciberagresiones a través del teléfono móvil y de Internet

\begin{tabular}{|c|c|c|c|c|c|}
\hline & & & & $(N=1390)$ & $\begin{array}{c}\text { Nunca } \\
954(68.6 \%)\end{array}$ \\
\hline \multicolumn{6}{|c|}{ Duración } \\
\hline $\begin{array}{l}\text { Ciberagresiones } \\
\text { Intensidad }\end{array}$ & $\leq 1$ mes & 3-6 meses & 6-12 meses & $\geq 1$ año & Total \\
\hline Moderada & $307(22.1 \%)$ & $40(2.9 \%)$ & $20(1.4 \%)$ & $1(0.1 \%)$ & $368(26.5 \%)$ \\
\hline Severa & $28(2.0 \%)$ & $20(1.4 \%)$ & $16(1.2 \%)$ & $4(0.3 \%)$ & $68(4.9 \%)$ \\
\hline Total & $335(24.1 \%)$ & $60(4.3 \%)$ & $36(2.6)$ & $5(0.4 \%)$ & $1390(100.0 \%)$ \\
\hline
\end{tabular}

Intensidad-duración ciberagresiones: $r=-.85, p<.01$ 
Tabla 2. Diferencias entre género en los ítems de ciberagresiones

\begin{tabular}{|c|c|c|c|c|c|}
\hline & $F$ & $p$ & $\eta^{2}$ & Contrastes & Medias \\
\hline \multicolumn{6}{|l|}{ Hostigamiento } \\
\hline $\begin{array}{l}\text { 1. He insultado o ridiculizado a alguien } \\
\text { con mensajes o llamadas } \\
\text { 8. He dicho o enviado cosas de miedo o } \\
\text { cosas guarras para asustar o fastidiar a alguien }\end{array}$ & $\begin{array}{l}F(1,1390)=12.12 \\
F(1,1390)=9.13\end{array}$ & $<.001$ & $\begin{array}{l}.009 \\
.007\end{array}$ & $\begin{array}{l}\mathrm{V}>\mathrm{M} \\
\mathrm{V}>\mathrm{M}\end{array}$ & $\begin{array}{l}1.66>1.49 \\
1.27>1.16\end{array}$ \\
\hline \multicolumn{6}{|l|}{ Persecución } \\
\hline $\begin{array}{l}\text { 2. He obligado a hacer cosas con amenazas } \\
\text { (traer dinero, hacer trabajos, sexo) } \\
\text { 7. He amenazado para meterme miedo }\end{array}$ & $\begin{array}{l}F(1,1390)=4.50 \\
F(1,1390)=6.44\end{array}$ & $\begin{array}{l}.034 \\
.011\end{array}$ & $\begin{array}{l}.003 \\
.005\end{array}$ & $\begin{array}{l}V>M \\
V>M\end{array}$ & $\begin{array}{l}1.12>1.07 \\
1.31>1.22\end{array}$ \\
\hline \multicolumn{6}{|l|}{ Denigración } \\
\hline $\begin{array}{l}\text { 4. He contado mentiras o rumores } \\
\text { falsos sobre alguien }\end{array}$ & $F(1,1390)=2.93$ & .087 & .012 & & \\
\hline \multicolumn{6}{|l|}{ Violación de la intimidad } \\
\hline $\begin{array}{l}\text { 5. He contado secretos de otro para fastidiarle } \\
\text { 6. He pasado y/o manipulado fotos o } \\
\text { videos de alguien sin su permiso } \\
\text { 9. Me he metido en el messenger o en cuentas } \\
\text { privadas de otros sin que pueda hacer nada }\end{array}$ & $\begin{array}{l}F(1,1390)=0.12 \\
F(1,1390)=5.50 \\
F(1,1390)=2.71\end{array}$ & $\begin{array}{l}.726 \\
.019\end{array}$ & $\begin{array}{r}<.001 \\
.004 \\
.002\end{array}$ & $\mathrm{~V}>\mathrm{M}$ & $1.22>1.14$ \\
\hline \multicolumn{6}{|l|}{ Exclusión Social } \\
\hline $\begin{array}{l}\text { 3. He hecho llamadas y no he contestado o } \\
\text { he dicho de conectarse y no he respondido }\end{array}$ & $F(1,1390)=1.24$ & .265 & .001 & & \\
\hline \multicolumn{6}{|l|}{ Suplantación de la Identidad } \\
\hline $\begin{array}{l}\text { 10. Me he hecho pasar por otro para decir o } \\
\text { hacer cosas malas por el móvil o en internet }\end{array}$ & $F(1,1390)=0.40$ & .488 & $<.001$ & & \\
\hline
\end{tabular}

$F$ : F de Snedecor, $p$ : nivel de significación, $\eta^{2}$ : Tamaño del efecto, contraste: comparaciones significativas, M: Mujeres, V: Varones

Media (desviación típica) en los ítems de ciberagresiones según sexo

\begin{tabular}{ccccccccccc}
\hline & Item & & & & & & & & & \\
Sexo & 1 & 8 & 2 & 7 & 4 & 5 & 6 & 9 & 3 & 10 \\
\hline Varón & $1.66(.96)$ & $1.27(.76)$ & $1.12(.46)$ & $1.31(.75)$ & $1.48(.81)$ & $1.31(.71)$ & $1.22(.68)$ & $1.37(.86)$ & $1.39(.81)$ & $1.25(.73)$ \\
Mujer & $1.49(.87)$ & $1.16(.54)$ & $1.07(.37)$ & $1.22(.62)$ & $1.40(.78)$ & $1.30(.69)$ & $1.14(.55)$ & $1.30(.75)$ & $1.44(.86)$ & $1.202(.65)$ \\
\hline
\end{tabular}

otros para fastidiarle» $(F(3,1390)=2.76, p<.05)$, «He pasado y/o manipulado fotos o videos de alguien sin su permiso $\rangle(F(3,1390)=4.13, p<.01)$ y $\langle$ Me he metido en el messenger o en cuentas privadas de otros $\sin$ que pueda hacer nada $>(F(3,1390)=3.03, p<$ $.05)$.

También en la agresión de suplantación de la identidad, «Me he hecho pasar por otro para decir o hacer cosas malas por el móvil o en internet > $(F(3,1390)=$ 3.64, $p<.05)$, los alumnos de $4^{\circ}$ de la ESO puntúan más alto que los de $3^{\circ}$ y también que los de $2^{\circ}$ de la ESO. Asimismo, las puntuaciones son estadísticamente más elevadas en $4^{\circ}$ de la ESO, con respecto a todos los cursos de Educación Secundaria Obligatoria en la cyberagresión de exclusión social «He hecho llamadas y no he contestado o he dicho de conectarse y no he respondido $>(F(3,1390)=7.46, p<.001)$. En la agresión de hostigamiento, « $<$ He insultado o ridiculizado a alguien con mensajes o llamadas», aparecen diferencias estadísticamente significativas entre $4^{\circ}$ de la ESO y $1^{\circ}$ de la $\operatorname{ESO},(F(3,1390)=2.71, p<.05)$.

\section{Discusión}

En este estudio se planteó como objetivo analizar la prevalencia de agresores a través de las nuevas tecnologías de la información y de la comunicación (teléfono móvil y internet), teniendo en cuenta en este análisis la duración y intensidad del acoso. También se exa- 
Tabla 3 Diferencias entre cursos en los ítems de ciberagresiones

$\begin{array}{lllll}F & p & \eta^{2} & \text { Contrastes } & \text { Medias }\end{array}$

Hostigamiento

1. He insultado o ridiculizado a alguien con mensajes o llamadas

8. He dicho o enviado cosas de miedo o cosas guarras para asustar o fastidiar a alguien

$\begin{array}{lllll}F(3,1390)=2.71 & .044 & .006 & 4^{\circ}>1^{\circ} & 1.69>1.51 \\ F(3,1390)=1.07 & .384 & .002 & & \end{array}$

\title{
Persecución
}

2. He obligado a hacer cosas con amenaza (traer dinero, hacer trabajos, sexo)

7. He amenazado a alguien para meterle miedo

$$
F(3,1390)=1.36 \quad .253 \quad .003
$$

$F(3,1390)=1.41 \quad .238 \quad .003$

\section{Denigración}

4. He contado mentiras o rumores falsos sobre alguien

\section{Violación de la intimidad}

5 .He contado secretos de otro para fastidiarle

6. He pasado y/o manipulado fotos o videos de alguien sin su permiso

9. Me he metido en el messenger o en cuentas privadas de otros sin que pueda hacer nada

$$
\begin{aligned}
& F(3,1390)=2.76 \\
& F(3,1390)=4.13 \\
& F(3,1390)=3.03
\end{aligned}
$$

.028

$4^{\circ}>3^{\circ}$

$1.39>1.24$

.009

$4^{\mathrm{o}}>3^{\mathrm{o}}$

$1.28>1.11$

Exclusión Social

3. He hecho llamadas y no he contestado o he dicho de conectarse y no he respondido

\section{Suplantación de la Identidad}

10. Me he hecho pasar por otro para decir o hacer cosas malas por el móvil o en internet

\author{
$F(3,1390)=3.64$
}

.01

$4^{\circ}>2^{\circ}$
$4^{\circ}>3^{\circ}$

$1.34>1.20$ $1.34>1.18$

\begin{tabular}{|c|c|c|c|c|c|c|c|c|c|c|}
\hline & Item & & & & & & & & & \\
\hline Curso & 1 & 8 & 2 & 7 & 4 & 5 & 6 & 9 & 3 & 10 \\
\hline $1^{\circ} \mathrm{ESO}$ & $1.51(.95)$ & $1.22(.75)$ & $1.12(.54)$ & $1.26(.77)$ & $1.41(.86)$ & $1.26(.62)$ & $1.16(.62)$ & $1.29(.81)$ & $1.34(.84)$ & $1.22(.73)$ \\
\hline $2^{\circ} \mathrm{ESO}$ & $1.60(.93)$ & $1.25(.74)$ & $1.10(.41)$ & $1.26(.66)$ & $1.45(.77)$ & $1.33(.75)$ & $1.16(.66)$ & $1.35(.82)$ & $1.41(.82)$ & $1.20(.64)$ \\
\hline $3^{\circ} \mathrm{ESO}$ & $1.53(.86)$ & $1.16(.53)$ & $1.05(.26)$ & $1.22(.68)$ & $1.36(.58)$ & $1.24(.44)$ & $1.11(.67)$ & $1.29(.71)$ & $1.29(.56)$ & $1.18(.56)$ \\
\hline $4^{\circ} \mathrm{ESO}$ & $1.69(.92)$ & $1.22(.58)$ & $1.10(.40)$ & $1.33(.73)$ & $1.54(.84)$ & $1.39(.74)$ & $1.28(.32)$ & $1.44(.88)$ & $1.59(.92)$ & $1.34(.79)$ \\
\hline
\end{tabular}

F: F de Snedecor, $p$ : nivel de significación, ${ }^{2}$ : Tamaño del efecto, contrastes: comparaciones significativas, Cursos de Enseñanza Secundaria Obligatoria: $1^{\circ}: 1^{\circ}$ Eso, $2^{\circ}: 2^{\circ}$ Eso, $3^{\circ} ; 3^{\circ}$ Eso, $4^{\circ}: 4^{\circ}$ Eso

Media (desviación típica) en los ítems de ciberagresiones según curso

minaron las diferencias de género y de curso en función de los tipos de agresiones tecnológicas cometidas por el adolescente.

Los resultados de nuestro trabajo indicaron que en el último año, el $68.6 \%$ de los adolescentes no han acosado a sus iguales a través de las nuevas tecnologías. Los resultados sugieren, por tanto, que la prevalencia de cyberbullies es del $26.5 \%$ para el acoso moderado, y del $4.9 \%$ para el acoso severo. Estos resultados sobre la prevalencia de agresores cibernéticos entre adolescentes concuerda con el trabajo de Smith et al. (2006), y también van en la línea, aunque con menor incidencia, a los obtenidos por Calvete et al. (2010) con adolescentes españoles. Ya ha sido mencionada y discutida la dificultad para comparar los resultados de los estudios nacionales e internacionales debido a la diver- 
sidad de metodologías y medidas utilizadas entre los trabajos. En nuestra investigación, se evalúa un amplio conjunto de conductas de acoso cibernético, por lo que los datos referidos a las diferencias de género y de curso, proporcionan además, de una información novedosa, un conocimiento más exhaustivo y completo sobre los diferentes tipos de conductas de cyberbullying, que tienen según su mayor o menor potencial dañino, consecuencias más graves sobre el ajuste psicosocial de la víctima.

En lo que respecta a las diferencias de género, de las diez conductas de acoso cibernéticas evaluadas, nuestros datos sugieren que en la mitad de estas ciberagresiones, la prevalencia es mayor entre los chicos, mientras que en las otras cinco conductas no se observan diferencias estadísticamente significativas entre sexos.

En concreto, nuestros resultados indican que los chicos acosan más en conductas cibernéticas directas relativas a acciones de hostigamiento y persecución, tales como molestar o amenazar mediante comunicaciones directas con la víctima -aún jugando con la "ventaja" del anonimato-, y de difusión de imágenes degradantes para la víctima. Estos resultados son consistentes con los hallazgos obtenidos en recientes investigaciones (Garmendia et al., 2011; Kowalski et al., 2010; Li, 2007; Navarro, 2009; Ortega et al., 2008; Sourander et al., 2010). Más en concreto, Calvete et al. (2010) encuentran, como en nuestra investigación, que los chicos presentan una mayor tendencia que las chicas en enviar contenido sexual no deseado, y en grabar y difundir imágenes degradantes sobre la víctima. Este resultado va en la línea de lo que ocurre también en el acoso escolar, dónde los chicos protagonizan más agresiones directas y físicas y las chicas, agresiones indirectas y relacionales (Almeida, 1999; Buelga, Musitu, Murgui y Pons, 2008; Buelga et al., 2010; Cava, 2011; Tapper y Boulton, 2004).

De hecho, nuestros resultados indican que en el caso del cyberbullying, no hay diferencias de género en cuanto al sexo de los agresores en conductas cibernéticas más indirectas y relacionales que no suponen una comunicación directa con la víctima, sino con terceros. Tanto chicos como chicas acosarían por igual a sus pares en agresiones de denigración (difusión de mentiras y rumores sobre el otro), violación de la intimidad (difusión de información confidencial, intromisión en cuentas privadas), suplantación de la identidad y exclusión social. Sin embargo, en el rol de víctima, sí que aparecen diferencias significativas entre sexos en este tipo de agresiones cibernéticas relacionales. El trabajo de Buelga et al. (2010) pone de manifiesto que las chicas están más victimizadas en este tipo de agresiones cibernéticas que los chicos.

Por otra parte, un resultado sorprendente del presente trabajo tiene que ver con la edad de los cyberbullies, que indica que aumentan con respecto a cursos inferiores de enseñanza secundaria obligatoria, las ciberagresiones en $4^{\circ}$ curso (15- 16 años). Las diferencias estadísticamente significativas se hallan en concreto, entre $4^{\circ}$ y $3^{\circ}$ de la ESO, lo que sugiere que, en general, en $3^{\circ}$ hay un ligero descenso de ciberagresiones respecto a $1^{\circ}$ y $2^{\circ}$, con un claro ascenso en $4^{\circ}$ en las ciberagresiones en esta edad.

Trabajos como los de Li (2007), Kowalski y Limber (2007), Smith et al. (2008) o Worthen (2007) sugieren que el cyberbullying es más frecuente en la adolescencia temprana, observándose una disminución en la adolescencia media. Nuestros resultados, sin embargo, muestran que las conductas de ciberagresiones aumentan en la adolescencia media. Las ciberagresiones más frecuentes entre los adolescentes de $4^{\circ}$ con respecto a los de $3^{\circ}$ son la divulgación de rumores sobre la víctima, violación de la intimidad: divulgación de secretos, intromisión en cuentas privadas y difusión de imágenes vejatorias, suplantación de la identidad, y conductas de hostigamiento y persecución.

De cualquier forma, las investigaciones sobre este tema no son concluyentes. De hecho, Ortega et al. (2008) no hallan diferencias significativas entre edades en la conducta del ciberagresor, aunque sí observan que la mayoría de los acosadores están en los dos últimos cursos de Educación Secundaria Obligatoria. Por otro lado, Calvete et al. (2010) encuentran que en segundo y tercer curso hay una mayor incidencia de conductas de ciberacoso que en primer y cuarto cursos. De cualquier modo, deberá investigarse más para estimar la incidencia que sobre los resultados de la investigación tienen las diferentes escalas de medición, la procedencia geográfica y cultural de las muestras, así como la evolución de esta conducta a través del tiempo.

Habrá que ahondar también en la comprensión de nuevas cuestiones sobre un tema novedoso y aún con muchos interrogantes por resolver y aspectos por descubrir. A este respecto, algunos estudios, como Li (2006), encuentran una correlación entre el rol de cibervíctima y el de ciberagresor. El cyberbullying es similar a otras formas de bullying salvo en una diferencia fundamental: la tecnología separa físicamente a la víctima del agresor, lo que podría permitir que adolescentes tímidos acosaran a otros, a veces en represalia por actos previos de bullying o de cyberbullying (Berger, 2007; Martínez, Moreno, Amador y Orford, 2011). Del mismo modo, como señala Li (2007), algunas cibervíctimas son miembros de grupos de iguales en los que la reiteración de bromas a través de medios tecnológicos se convierte en norma, por lo que entran en una espiral de victimización-agresión con sus propios amigos. Siguiendo a Calvete et al. (2010), es posible que algunos adolescentes que no se comportarían de forma agresiva en una situación cara a cara puedan hacerlo a través de un medio tecnológico, generándose el interrogante de si el perfil psicológico de los adolescentes que llevan a cabo cyberbullying es similar o diferente al perfil asociado a las formas tradicionales de acoso entre iguales. Estas cuestiones nos llevan a 
considerar, con Stephenson y Smith (1989) y Tokunaga (2010), que un requisito previo para que una situación sea considerada acoso entre iguales es la inclusión de una intención intimidadora que provoque distrés en la víctima.

El aumento de este problema del cyberbullying requiere que las escuelas, las familias, los agentes comunitarios y las instituciones se impliquen en desarrollar acciones que prevengan, controlen y regulen las agresiones tecnológicas entre iguales (Walker, 2010). La investigación sobre este fenómeno es una prioridad en el ámbito social y educativo. Con el actual uso generalizado de los medios tecnológicos de comunicación social por los adolescentes y su efecto sobre el acoso entre iguales, ese esfuerzo debería ser prioritario para los educadores y los investigadores sociales y de la educación.

Como cualquier trabajo científico, este estudio no está exento de limitaciones. En primer lugar, es importante reseñar que los resultados expuestos en este trabajo deben interpretarse con precaución, debido a la naturaleza transversal del mismo, por lo que un estudio longitudinal con medidas en distintos tiempos ayudaría a la confirmación de los resultados aquí observados. Por otro lado, en nuestro trabajo las respuestas proporcionadas por los adolescentes han sido elaboradas a través de autoinformes y, aunque podría penarse que estuvieran sujetas a efectos de deseabilidad social y sesgos, como indican Flisher, Evans, Muller y Lombard (2004) la fiabilidad y validez de los autoinformes de los adolescentes en relación a la medición de conductas de riesgo resulta ser bastante aceptable. Los autoinformes presentan ventajas muy notables, que han generalizado su utilización en ciencias sociales, como permitir recoger información significativa en un período temporal corto y facilitar la interpretación de los datos sin reelaboraciones sujetas a inferencias. Algunos estudios sobre comportamientos de riesgo en la adolescencia han demostrado la validez de las medidas de autoinforme al compararlas con datos de fuentes alternativas (Kamphaus y Frick, 2005; Ritakallio, Kaltiala-Heino, Kivivuori y Rimpelä, 2005), pues los adolescentes son mucho más precisos en los informes sobre su propio comportamiento, ya que los padres o los profesores pueden ser desconocedores de la frecuencia y magnitud del comportamiento antisocial del hijo.

En definitiva, este trabajo proporciona observaciones novedosas sobre el fenómeno emergente del cyberbullying, en tanto que aporta datos acerca de la prevalencia de cyberbullies y de las diferencias de género y de curso en función de las agresiones cibernéticas, cuestiones escasamente estudiada en la literatura científica. De cualquier modo, como ha sido discutido a lo largo del trabajo, todavía son muchos los tópicos y temas que necesitan ser investigados, ya que aún se desconoce, en gran medida, la naturaleza y funcionamiento de este nuevo tipo de acoso. Un tipo de maltra- to entre iguales, propio de una sociedad tecnológica, cuyos jóvenes pertenecen a una nueva generación en la que los niños crecen relacionándose con y a través de medios cada vez más complejos, e interiorizando su uso y posibilidades como una parte de la vida cotidiana (Oblinger y Oblinger, 2005; Veen, 2003).

\section{Agradecimientos}

Este trabajo ha sido elaborado en el marco del Proyecto de Investigación PSI2008-01535/PSIC "Violencia escolar, victimización y reputación social en la adolescencia", subvencionado por el Ministerio de Ciencia e Innovación de España.

\section{Referencias}

Almeida, A. (1999). Bullying in Portugal. En P. K. Smith, Y. Morita, J. Junger-Tas, D. Olweus, R. Catalano y P. Slee (Eds.), The nature of school bullying: A cross-national perspective. Londres: Routlegde.

Berger, K.S. (2007). Update on bullying at school: Science forgotten? Developmental Review, 27, 90-126.

Bickham, D. S. y Rich, M. (2009). Global assessment of online threats and intervention opportunities for adolescents. Journal of Adolescent Health, 44(suppl.), 24-25.

Buelga, S., Cava, M. J. y Musitu, G. (2010). Cyberbullying: victimización entre adolescentes a través del teléfono móvil y de internet. Psicothema, 22, 784-789.

Buelga, S., Musitu, G. y Murgui, S. (2009). Relaciones entre la reputación social y la agresión relacional en la adolescencia. International Journal of Clinical and Health Psychology, 9, 127-141.

Buelga, S., Musitu, G., Murgui, S. y Pons, J. (2008). Reputation, loneliness, satisfaction with life and aggressive behavior in adolescence. The Spanish Journal of Psychology, 11, 192-200.

Burgess-Proctor, A., Patchin, J. W. y Hinduja, S. (2009). Cyberbullying and online harassment: Reconceptualizing the victimization of adolescent girls. En V. Garcia y J. Clifford (Eds.), Female crime victims: Reality reconsidered. Upper Saddle River: Prentice Hall.

Calvete, E., Orue, I., Estévez, A., Villardón, L. y Padilla, P. (2010). Cyberbullying in adolescents: Modalities and aggressors' profile. Computers in Human Behavior, 26, $1128-1135$.

Campbell, M. A. (2005). Cyberbullying: An old problem in a new guise? Australian Journal of Guidance and Counselling, 15, 68-76.

Cava, M. J. (2011). Familia, profesorado e iguales: claves para el apoyo a las víctimas de acoso escolar. Psychosocial Intervention, 20, 183-192.

Cava, M. J., Buelga, S., Musitu, G. y Murgui, S. (2010). Violencia escolar entre adolescentes y sus implicaciones en el ajuste psicosocial: un estudio longitudinal. Revista de Psicodidáctica, 15, 21-34. 
Cava, M. J., Musitu, G., Buelga, S. y Murgui, S. (2010). The relationships of family and classroom environments with peer relational victimization: An analyisis of their gender differences. The Spanish Journal of Psychology, 13, 156165.

David-Ferdon, C. y Feldman, M. (2007). Electronic media, violence and adolescents. Journal of Adolescent Health, 41(suppl.), 1-5.

Defensor del Pueblo (2007). Violencia escolar: El maltrato entre iguales en la Educación Obligatoria Secundaria. Madrid: Publicaciones de la Oficina del Defensor del Pueblo.

Dehue, F., Bolman, C. y Vollink, T. (2008). Cyberbullying: Youngsters' experiences and parental perception. Cyberpsychology and Behavior, 11, 217-223.

Didden, R., Scholte, R. H., Korzilius, H., De Moor, J. M., Vermeulen, A., O'Reilly, M. et al. (2009). Cyberbullying among students with intellectual and developmental disability in special education settings. Developmental Neurorehabilitation, 12, 146-151.

Finkelhor, D., Mitchell, K. J. y Wolak, J. (2000). Online victimization: A report on the nation's youth. Alexandría: National Center for Missing and Exploited Children.

Flisher, A. J., Evans, J., Muller, M. y Lombard, C. (2004). Brief report: Test-retest reliability of self-reported adolescent risk behaviour. Journal of Adolescence, 27, 207-212.

García-Pérez, R. (2011). Violencia y victimización en la adolescencia: La perspectiva de los adolescentes. Tesis Doctoral, Universidad de Sevilla.

Garaigordobil, M. (2011). Prevalencia y consecuencias del cyberbullying: una revisión. International Journal of Psychology and Psychological Therapy, 11, 233-254

Garmendia, M., Garitaonandia, C., Martínez-Fernández, G. y Casado, M. A. (2011). Riesgos y seguridad en internet: Menores españoles en el contexto europeo. Bilbao: Euskal Herriko Univertsitatea.

Genta, M. L., Brighi, A. y Guarini, A. (2009). European project on bullying and cyberbullying granted by Daphne II Programme. Journal of Psychology, 217, 233-234.

Gradinger, P., Strohmeier, D. y Spiel, C. (2009). Traditional bullying and cyberbullying: Identification of risk groups for adjustment problems. Journal of Psychology, 217, 205-213.

Hinduja, S. y Patchin, J. W. (2008). Cyberbullying: An exploratory analysis of factors related to offending and victimization. Deviant Behavior, 29, 129-156.

Huesmann, L. R. (2007). The impact of electronic media violence: Scientific theory and research. Journal of Adolescent Health, 41(suppl.), 6-13.

Juvoven, J. y Gross, E. F. (2008). Bullying experiences in cyberspace. The Journal of School Health, 78, 496-505.

Kamphaus, R. W. y Frick, P. J. (2005). Clinical assessment of child and adolescent personality and behavior. Nueva York: Springer.

Katzer, C., Fetchenhauer, D. y Belschak, F. (2009). Cyberbullying: Who are the victims? A comparison of victimization in internet chatrooms and victimization in school. Journal of Media Psychology, 21, 25-36.
Keith, S. y Martin, M. E. (2005). Cyberbullying: Creating a culture of respect in a cyber world. Reclaiming Children and Youth, 13, 224-228.

Kowalski, R. y Limber, S. P. (2007). Electronic bullying among middle school students. Journal of Adolescent Health, 41(suppl.), 22-30.

Kowalski, R., Limber, S. y Agatston, P. (2010). Cyberbullying: El acoso escolar en la era digital. Bilbao: Desclée de Brouwer.

Li, Q. (2007). New bottle but old wine: A research of cyberbullying in schools. Computers in Human Behavior, 23, $1777-1791$.

Li, Q. (2008). A cross-cultural comparison of adolescents' experience related to cyberbullying. Educational Research, 50, 223-234.

Martínez, B., Moreno, D, Amador, L. y Orford, J. (2011). School victimization among adolescents. An analysis from an ecological perspective, Psychosocial Intervention, 20, 149-160.

McKenna, P. (2007). The rise of cyberbullying. The New Scientist, 195, 26-27.

Monks, C. P., Smith, P. K., Naylor, P., Barter, C., Ireland, J. L. y Coyne, I. (2009). Bullying in different contexts: Commonalities, differences and the role of theory. Aggression and Violent Behavior, 14, 1359-1789.

Mora-Merchán, J. A. (2008). El cyberbullying: Un nuevo reto para la convivencia en nuestras escuelas. Informació Psicològica, 94, 60-70.

Nansel, T. R., Overpeck, M., Pilla, R. S., Ruan, W. J., Simons-Morton, B. y Scheidt, P. (2001). Bullying behaviors among US youth: Prevalence and association with psychosocial adjustment. Journal of the American Medical Association, 285, 2094-2100.

Navarro, R. (2009). Variables psicosociales de la agresión escolar: La variable género como factor diferencial. Tesis Doctoral, Universidad de Castilla - La Mancha.

Oblinger, D. y Oblinger, J. L. (2005). Educating the net generation. Washington: Educause.

Olweus, D. (2006). El acoso escolar: Una revisión general. En A. Serrano (Ed.), Acoso y violencia en la escuela. Barcelona: Ariel.

Ortega, R., Calmaestra, J. y Mora-Merchán, J. A. (2008). Cyberbullying. International Journal of Psychology and Psychological Therapy, 8, 183-192.

Patchin, J. y Hinduja, S. (2006). Bullies move beyond the schoolyard: A preliminary look at cyberbullying. Youth Violence and Juvenile Justice, 4, 148-169.

Raskauskas, J. y Stoltz, A. D. (2007). Involvement in traditional and electronic bullying among adolescents. Developmental Psychology, 43, 564-575.

Rigby, K. (2002). New perspectives on bullying. Londres: Kingsley.

Ritakallio, M., Kaltiala-Heino, R., Kivivuori, J. y Rimpelä, M. (2005). Brief report: Delinquent behaviour and depression in middle adolescence. A Finnish community sample. Journal of Adolescence, 28, 155-159.

Santos, J., Muñoz, A., Juez, P., y Cortiñas, P. (2003). Diseño de encuestas de estudio de mercado. Técnicas de mues- 
treo y análisis multivariente. Madrid: Fundación Ramón Areces.

Schultze-Krumbholz, A. y Scheithauer, H. (2009). Socialbehavioral correlates of cyberbullying in a German student sample. Journal of Psychology, 217, 224-226.

Smith, P. K, Mahdavi, J., Carvalho, C., Fisher, S., Russell, S. y Tippett, N. (2008). Cyberbullying: Its nature and impact in secondary school pupils. Journal of Child Psychology and Psychiatry, 49, 376-385.

Smith, P. K., Mahdavi, J., Carvalho, C. y Tippett, N. (2006). An investigation into cyberbullying, its forms, awareness and impact, and the relationship between age and gender in cyberbullying. Londres: Anti-Bullying Alliance.

Sourander, A., Brunstein-Klomek, A., Ikonen, M., Lindroos, J., Luntamo, T., Koskelainen, M. et al. (2010). Psychosocial risk factors associated with cyberbullying among adolescents: A population-based study. Archives of General Psychiatry, 67, 720-728.

Spears, B., Slee, P., Owens, L. y Johnson, B. (2009). Behind the scenes and screens: Insights into the human dimension of covert and cyberbullying. Journal of Psychology, 217, 189-196.

Stephenson, P. y Smith, D. (1989). Bullying in the junior school. En D. P. Tattum y D. A. Lane (Eds.), Bullying in schools. Stoke-an-trent: Trentham Books.

Subrahmanyam, K. y Greenfield, P. (2008). Online communication and adolescent relationships. The Future of Children, 18, 119-146.

Tapper, K. y Boulton, M. J. (2004). Sex differences in levels of physical, verbal and indirect aggression amongst pri- mary school children and their associations with beliefs about aggression. Aggressive Behavior, 30, 123-145.

Tokunaga, R. S. (2010). Following you home from school: A critical review and synthesis of research on cyberbullying victimization. Computers in Human Behavior, 26, $277-$ 287.

Veen, W. (2003). A new force for change: Homo zappiens. The Learning Citizen, 7, 5-7.

Walker, H. M. (2010). Relational aggression in schools: Implications for future research on screening intervention and prevention. School Psychological Review, 39, 594-601.

Willard, N. E. (2006). Cyberbullying and cyberthreats: Responding to the challenge of online social aggression, threats and distress. Eugene: Center for Safe and Responsible Internet Use.

Willard, N. E. (2007). The authority and responsibility of school officials in responding to cyberbullying. Journal of Adolescent Health, 41(suppl.), 64-65.

Williams, K. R. y Guerra, N. G. (2007). Prevalence and predictors of internet bullying. Journal of Adolescent Health, 41(suppl.), 14-21.

Wolak, J., Mitchell, K. y Finkelhor, D. (2006). Online victimization: Five years later. Alexandría: National Center for Missing and Exploited Children.

Worthen, M. (2007). Education policy implications from the expert panel on electronic media and youth violence. Journal of Adolescent Health, 41(suppl.), 61-63.

Ybarra, M. (2004). Linkages between depressive symptomatology and internet harassment among young regular internet users. Cyberpsychology and Behavior, 7, 247-257.

Manuscrito recibido: 21/07/2011

Revisión recibida: 18/10/2011

Manuscrito aceptado: 04/11/2011 